"NOTICE: this is the author's version of a work that was accepted for publication in NDT \& E International. Changes resulting from the publishing process, such as peer review, editing, corrections, structural formatting, and other quality control mechanisms may not be reflected in this document. Changes may have been made to this work since it was submitted for publication. A definitive version was subsequently published in NDT \& E International, [VOL 66, September 2014] DOI: 10.1016/j.ndteint.2014.05.001

The final version can be found here (http://www.sciencedirect.com/science/article/pii/S0963869514000711) 


\title{
Subsurface Cracks in AGR Graphite Bricks
}

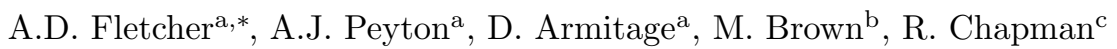 \\ ${ }^{a}$ School of Electrical and Electronic Engineering, University of Manchester, UK, M13 9PL \\ ${ }^{b}$ EDF Energy, Barnett Way, Barnwood, Gloucester, UK, GL4 3RS \\ ${ }^{c}$ NDT Consultant, Gloucester, UK, GL11 $4 \mathrm{EW}$
}

\begin{abstract}
Sub-surface cracks present difficulties for eddy current methods as the skin effect causes currents to flow preferentially near the surface. This work examines graphite as a case study for the qualitative assessment of sub-surface cracks and employs multi-frequency eddy current techniques. The research has particular relevance to Advanced Gas-Cooled Reactors (AGR's) as during their operation there is the potential for cracks to develop within moderator bricks. This work reports that subsurface slots of $18 \%$ brick thickness can be detected. This work confirms these results with a parametric 3D finite element study.
\end{abstract}

\section{Introduction}

One of the main aims of eddy current NDT technologies has been to develop methods by which defects in materials (particularly cracks) can be assessed quantitatively. An in-service inspection (ISI) of structural plant components will typically aim to determine the existence (and possible location) of a defect subject to certain sensitivity requirements. However, in order to ensure that the component will conform to structural requirements an assessment must be made of the component's integrity: that is the dimensions of the defect must be determined. Naturally, the major focus of work in this area has been on metals (see, for example [1, 2]).

Typically, an ultrasonic method will be used to make a qualitative assessment of material defects. However there are a number of situations in which this technology is unsuitable. In particular, the core of a nuclear reactor presents an environment which is hostile to all but the most robust methods. The high doses of radiation prevent the use of any unshielded electronic components and the necessary use of remote inspection equipment inhibits the use of a couplant (when this is necessary). Therefore, in order to make qualitative assessments of defects in these environments non-contact electromagnetic methods are preferred.

The Advanced Gas-Cooled Reactor (AGR) is the dominant design of commercial nuclear plant in the UK. Like the preceding MAGNOX reactors, it is a gas cooled, graphite moderated design. The moderator is made from a regular lattice of graphite blocks which serve three primary functions: to moderate fission neutrons; to act as a

\footnotetext{
*Corresponding Author

Email address: adam.fletcher-3@postgrad.manchester.ac.uk (A.D. Fletcher)
}

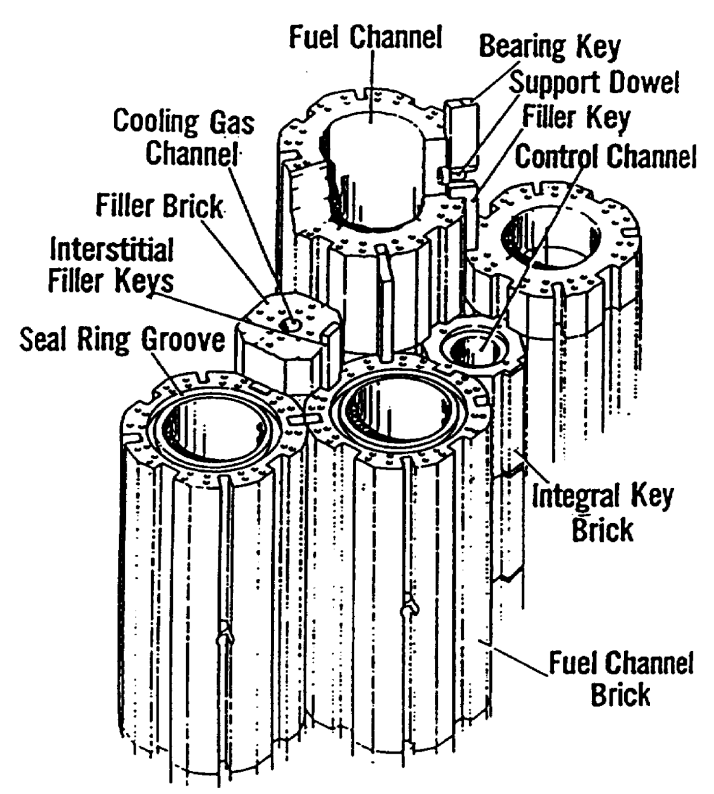

Figure 1: An isometric view of a typical the AGR core showing the fuel channel and interstitial bricks and the keying arrangement [3].

structural component which maintains the lattice spacing of fuel elements and control rods; and to act as a heat sink for the transfer of thermal energy to the primary coolant. This lattice is shown in Figure 1.

Cracking can occur because graphite undergoes dimensional change under irradiation, the rate of which is dependent on the neutron flux. At low doses, AGR graphite undergoes a volume reduction, but once a critical dose has been exceeded the graphite will begin to expand. Differential volume changes over the brick cross section lead to the development of strain fields over the brick.

These strain fields can ultimately lead to cracking within the bricks. In early life, the stresses at the bore dominate, 


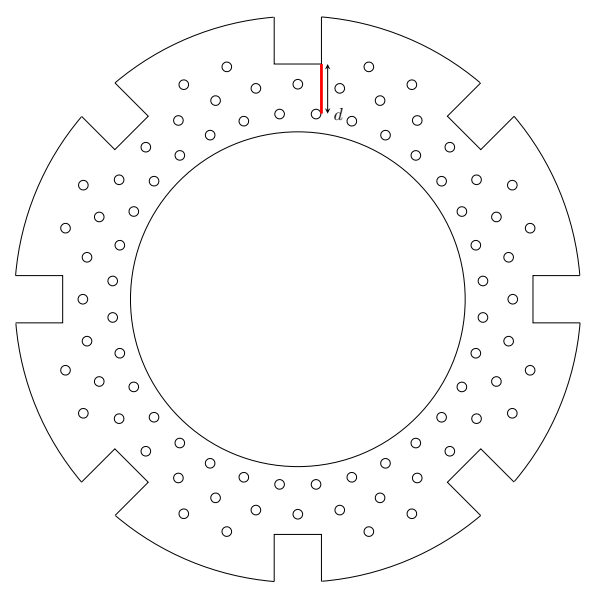

Figure 2: Plan view of an AGR fuel channel brick (Hartlepool/Heysham 1 type). The bricks used in this work had a bore diameter of $270 \mathrm{~mm}$. The keyway to bore distance is approximately $56 \mathrm{~mm}$. Also shown is a slot originating from a keyway root (red feature), of the type studied in this work. $d$ labels the radial size of the slot.

leading to radial cracking from the bore outwards to the periphery. In later life it is possible that the stresses at the periphery begin to dominate and could lead to cracks propagating inwards. The geometry of the brick (see Figure 2) suggests that in later life stresses are concentrated around keyway root corners and hence these are a likely site for crack initiation.

At present there is limited data on the number and extent of subsurface keyway cracks, if they occur at all. However, the safety implications of subsurface cracks may be severe and as part of the ongoing life extension programme it is important to understand the extent of this problem. Eddy current techniques have already been adapted to crack detection in graphite, though the present system has only been tested in-core on known bore breaking cracks. Work by EDF Energy suggests that subsurface machined slots of approximately $50 \%$ through wall extent may be detectable [4].

A great deal of work has been carried out on eddy current NDT problems as applied to nuclear plant components, however the majority of this is also focused on metals (for example $[5,6,7,8]$ ). This work presents graphite as a case study for the quantitative evaluation of subsurface cracks within a nuclear environment. At present machined slots have been used as an analogue for cracks so that the dimension of the defect can be reliably controlled. As a material for electromagnetic inspection graphite has a number of interesting properties.

Graphite has a conductivity which may be as much as three orders of magnitude lower than that found in metals and hence the penetration depth of electromagnetic fields into graphite is much higher (for a given excitation frequency) than in metals for a sample of comparable size. Furthermore, the components under consideration have a

\begin{tabular}{|c|c|}
\hline Region & Maximum Edge Length [mm] \\
\hline Slots & 5 \\
\hline Bore & 20 \\
\hline Layers 1-3 & 10 \\
\hline Layers 4-7 & 20 \\
\hline Layers 8-12 & 40 \\
\hline Layers 13-16 & 80 \\
\hline Layers 17-20 & 160 \\
\hline
\end{tabular}

Table 1: The meshing parameters used in the FE work. The brick layers are numbered from the bore radially outwards to the periphery.

thickness much greater than that typically considered for metal inspection problems and so a much larger sensor geometry is also required.

Despite its widespread use in both nuclear and nonnuclear applications, the study of inspection problems on graphite has been quite limited, with the majority of workers focusing on composites such as graphite-epoxy $[9,10]$ or as a component of cast iron [11]. Examinations of nuclear grade graphites have tended to focus on surface breaking defects and bulk resistivity estimation [12].

Furthermore, high grade graphite is the material of choice for the moderator of next generation high temperature gas-cooled reactors and so the experience of NDT methods applied to this material will be directly relevant for the inspection of future nuclear plants.

\section{Modelling}

The AGR core is made up of graphite bricks arranged on a square lattice. This work focuses on inspection of fuel channel bricks since these are most susceptible to cracking due to their proximity to the fuel. These bricks are machined with a number of features which makes modelling more difficult. The bricks have a series of vertical keyways machined around their outer face which support and stabilise the lattice structure. In addition each brick also contains an array of axial "methane holes" which improves the flow of coolant to the centre of the brick (see Figure 2).

An additional refinement has been to discretise the brick into annular layers. This allows for manual control of the mesh size within each layer. It was necessary to increase the density (decrease the maximum length) of tetrahedra closest to the brick bore. This ensured that the regions which contributed most strongly to the solution accurately reflected the geometry of that region. The maximum tetrahedra lengths used in this work are given in Table 1.

Throughout the modelling work only 2 dimensional radial slots were simulated. These were used to represent radially directed crack-like defects. This was achieved by inserting an insulating boundary into the geometry which creates a slot-like defect (see Figure 3). This slot originated from a keyway corner and was divided into segments of $5 \mathrm{~mm}$ radial extent to allow for the effective slot extent to be controlled. Three slots were simulated of 50, 30 and 


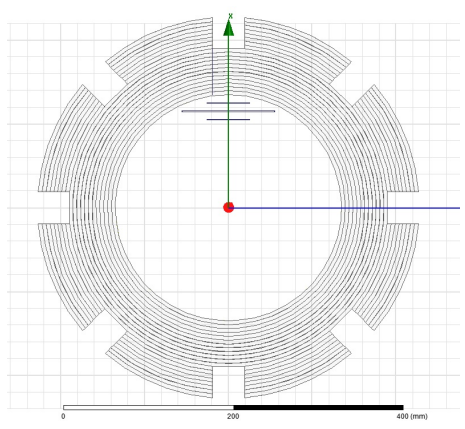

(a) Plan view. The slot is modelled as a series of $2 \mathrm{D}$ planar elements which originate from the left hand corner of the keyway at 12 o'clock.

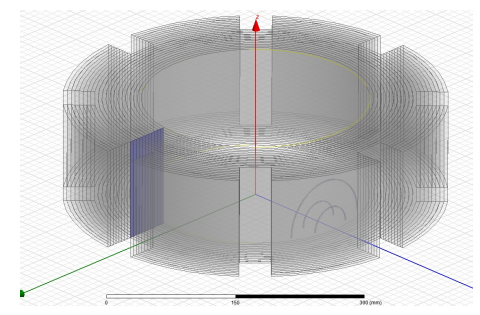

(b) Isometric view. Note that the horizontal symmetry plane has been exploited to reduce the number of elements.

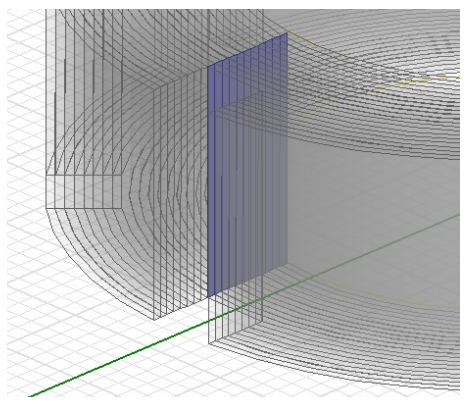

(c) Slot defect

Figure 3: The geometry used in the finite element modelling.
$10 \mathrm{~mm}$ through wall extent as measured along the slot face between the keyway corner and the slot tip. The $2 \mathrm{D}$ planes representing the slot were also controlled during the meshing, such that the edges of elements on these planes could not have a length greater than $5 \mathrm{~mm}$. All simulated slots were the full axial height of the brick.

Throughout the modelling work a gradiometer sensor has been used. This consisted of an identical pair of receiver coils of $50 \mathrm{~mm}$ diameter arranged equidistantly on either side of a $110 \mathrm{~mm}$ diameter exciter coil. The distance between each receiver and the exciter was $10 \mathrm{~mm}$ and the lift-off distance between the centre of the closest receive coil and the brick bore was $9.5 \mathrm{~mm}$. The coils were modelled as annular cylinders with a height and thickness of $0.5 \mathrm{~mm}$. The exciter was driven with a $1 \mathrm{~A}$ sinusoidal current and the mutual impedance between the receivers was calculated at each frequency.

The gradiometer position was varied between $-90^{\circ}$ and $90^{\circ}$ (where the origin was taken at the centre of the keyway containing the slot) and varied in $6^{\circ}$ increments. For each position, the excitation frequency was varied between $100 \mathrm{~Hz}$ and $100 \mathrm{kHz}$ and two simulations were performed using the same mesh: one containing the slot and one in which the insulating boundary condition was disabled to replicate a slot free brick.

The results of these simulations are shown in Figure 4 and Figure 5. These figures display the phase angle and amplitude of the mutual impedance between the receive coils for the frequency range $100 \mathrm{~Hz}$ to $100 \mathrm{kHz}$ and for coil positions between $-90^{\circ}$ and $90^{\circ}$. As can be seen, the phase change due to each slot can be observed when the difference in phase between the slotted and slot-free cases is plotted. As expected, the magnitude of the phase change increases with increasing slot size and the effect is localised with coil angle enabling the position of the slot to be determined. These figures also show that the background signal can be removed effectively. When the coil is a long way from the slot the phase change is reliably calculated to be zero, as would be expected from physical arguments. Also, it is observed that despite the fact that the geometry is remeshed between each coil position the change in phase angle with coil angle is relatively smooth. This gives additional evidence that the simulation is converging at each coil position and that the mesh is being well distributed over the angular region perturbed by the eddy currents. The response is also reasonably smooth with frequency. This is a more straightforward objective to achieve, however this observation does provide evidence that the mesh is well distributed over the thickness of the block, although it would appear that resolution is lost at higher frequencies suggesting that a greater mesh density may be needed at the bore of the brick. 


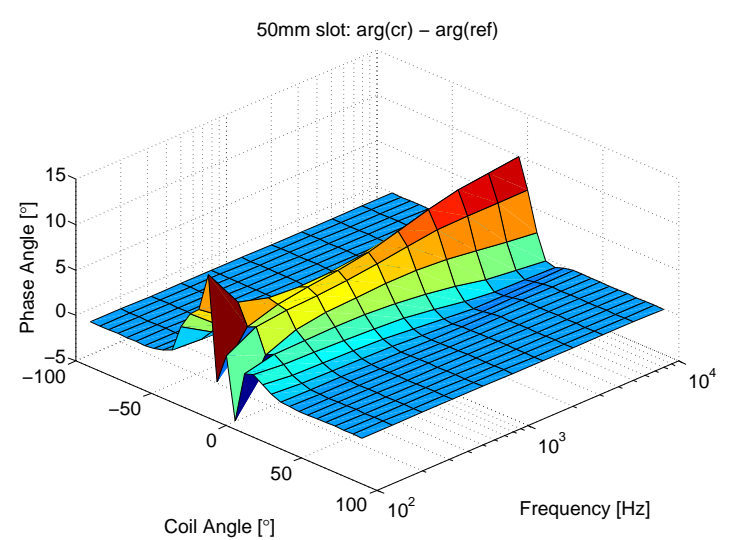

(a) $50 \mathrm{~mm}$ slot

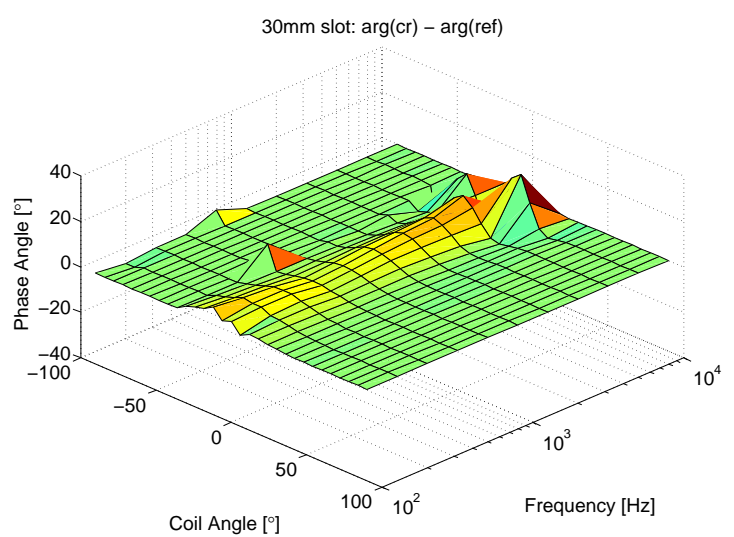

(b) $30 \mathrm{~mm}$ slot

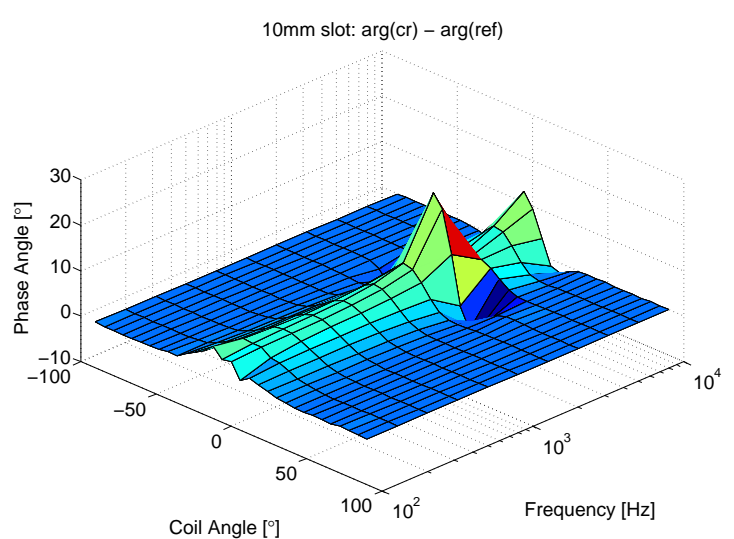

(c) $10 \mathrm{~mm}$ slot

Figure 4: Modelled phase changes for 3 radial slots (representing a crack-like defect). Calculated as $\arg ($ slotted) - $\arg$ (unslotted).

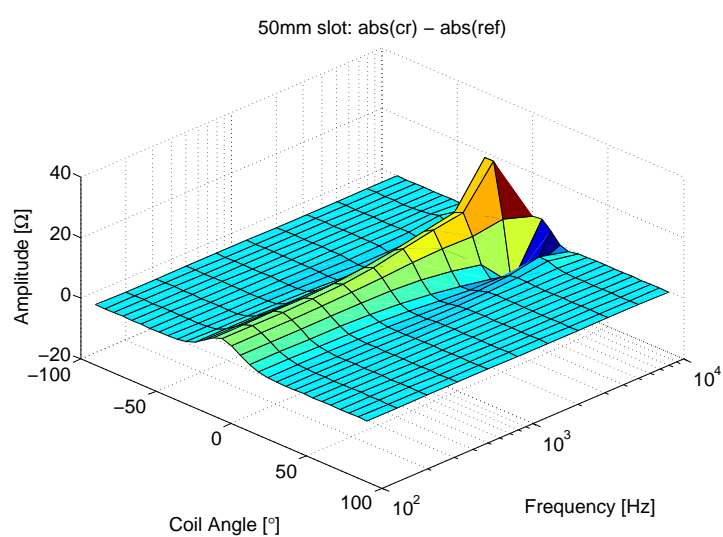

(a) $50 \mathrm{~mm}$ slot

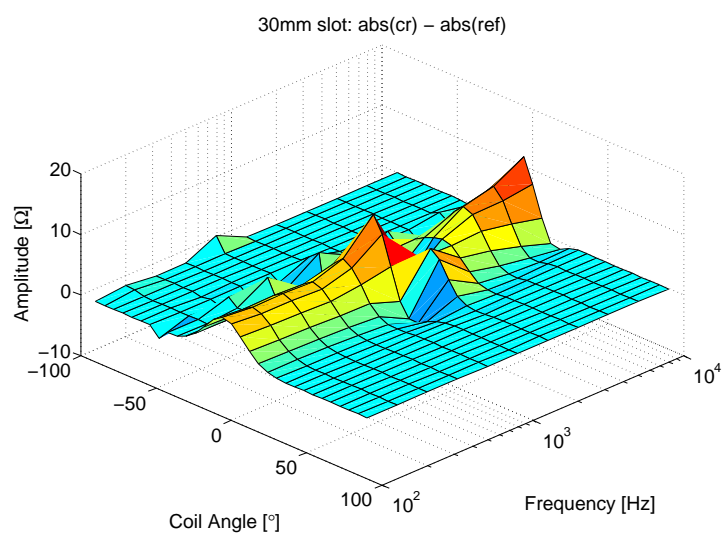

(b) $30 \mathrm{~mm}$ slot

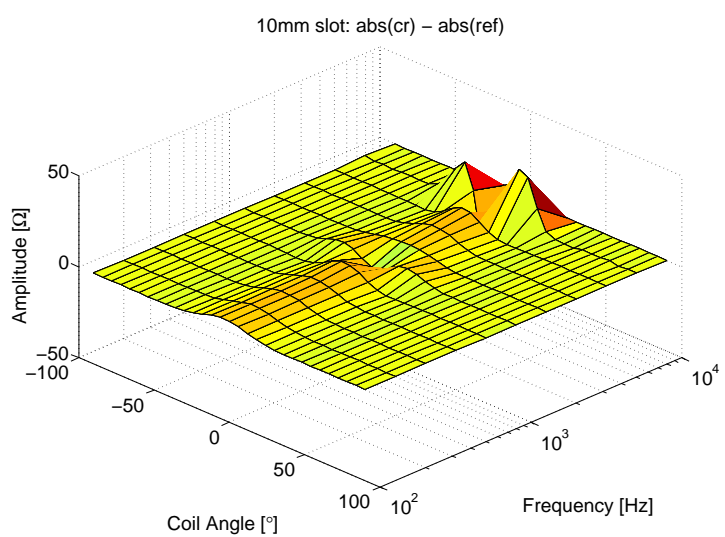

(c) $10 \mathrm{~mm}$ slot

Figure 5: Modelled amplitude changes for 3 radial slots (representing a crack-like defect). Calculated as abs(slotted) - abs(unslotted). 


\begin{tabular}{|l|c|c|}
\hline & $120 \mathrm{~mm}$ sensor & PECIT sensor \\
\hline $\begin{array}{l}\text { Mean exciter radius } \\
{[\mathrm{mm}]}\end{array}$ & 60 & 30 \\
\hline $\begin{array}{l}\text { Mean receiver ra- } \\
\text { dius [mm] }\end{array}$ & 30 & 26 \\
\hline Exciter turns & 150 & 178 \\
\hline Receiver turns & 200 & 356 \\
\hline $\begin{array}{l}\text { Receiver-Exciter } \\
\text { separation [mm] }\end{array}$ & 12 & 6 \\
\hline $\begin{array}{l}\text { Lift-off to first re- } \\
\text { ceiver [mm] }\end{array}$ & 11.5 & 7.5 \\
\hline
\end{tabular}

Table 2: Sensor geometries.

\section{Experimental}

\subsection{Methodology}

A number of AGR brick samples were provided by EDF Energy for use in this work. These were brick sections of $250 \mathrm{~mm}$ axial extent containing keyways and methane holes. Due to the limited performance of the finite element modelling in quantitatively resolving the response to a variable slot size an empirical approach was adopted.

This work used two gradiometer sensors: one has the same dimensions as used in the modelling work consisting of an exciter of 150 turns and each receiver of 200 turns; the other sensor is of the same geometry as that used on an AGR inspection tool (referred to as the PECIT sensor, Table 2). The gradiometer was located against the bore of the brick using a plastic stand-off and held in place with a sprung mount to ensure a constant lift-off. The frequency of excitation was swept between $100 \mathrm{~Hz}$ and $100 \mathrm{kHz}$ and the sensor impedance was measured at each frequency using a Solartron 1260 impedance analyser. The position of the coil was then displaced by a mechanical jig. Unfortunately, it was not possible to precisely control the angular coil displacement using this jig. Therefore, after a circumferential sweep was completed the data was interpolated to provide a fixed number of angular data points. Three complete circumferential sweeps were made and after interpolation the data taken from them was averaged.

The slot was then machined in $2 \mathrm{~mm}$ increments so that the radial extent of the slot was increased from $10 \mathrm{~mm}$ to $54 \mathrm{~mm}$ providing a dataset against which other defects can be sized. Each defect was measured with a vernier calliper after being cut. The width of the slot was held as constant and as small as the machining process would allow, between $2-3 \mathrm{~mm}$. This work has assumed that varying the width of the slot (ie: the separation between the slot faces) is negligible compared to the effect of varying the radial size of the slot. This is attributed to the fact that it is the cross sectional area of the slot (or crack) faces which disrupts the eddy current flow compared to the slot-free case allowing the slot to be detected. The slot width is very small compared to the other dimensions (at least five times smaller) and so once the slot is formed varying the width is assumed to have a minimal effect on the signal due to the defect.

It was found that the clearest indications of the cracks could be seen when the complex impedance was plotted in magnitude and phase components and that the phase component was particularly sensitive to the presence of slots. Figures 6 to 9 show the phase and amplitude responses measured for a sample of the slots after a positional average has been subtracted from them.

The plots of phase change with respect to coil position (Figures 6 and 7) appear to contain the most useful information. In each of these the location of the subsurface slots can be clearly observed and the maxima occur at the coil angle expected (ie: when the centre of the coil was closest to the slot tip). The plots of amplitude change with respect to coil position (Figures 8 and 9) do not appear to contain any useful information.

Examination of the frequency curves where the maximum phase change appears allows the approximate resolution of two sensors with respect to defect size to be determined. Plots of peak phase change with respect to the measured slot size are given in Figure 10. These figures demonstrate that radial slots down to $10 \mathrm{~mm}$ slant height can be detected and located using the phase component of receiver impedance of the $120 \mathrm{~mm}$ sensor and slots of more than approximately $20 \mathrm{~mm}$ slant height can be identified using the PECIT sensor (due to the higher level of noise at small slot sizes seen in the PECIT sensor).

The phase change undergoes a significant deviation from its background value when the gradiometer passes the slot. The peak in this trend occurs at a frequency between 0.8 and $1 \mathrm{kHz}$. This corresponds to a skin depth in the region of approximately $50-56 \mathrm{~mm}$ which is comparable with the bore-keyway distance, suggesting that the sensitivity of both sensors extends at least as far as the keyway root. This sensitivity will depend on not just the frequency of excitation but also the sensor size. A rough estimate would suggest that the sensitivity of the sensor would decrease rapidly when the depth of eddy current penetration (as measured by the skin depth) is much greater than the diameter of the coil. This is confirmed in that the maximum phase change measured increases rapidly as the slot size is increased (see Figure 10).

Figure 10 clearly shows that both the magnitude of the phase change and its frequency dependence are both strongly dependent upon the slot size. Also visible in this figure is the change in radial slot dependence which occurs as the slot begins to intersect the middle ring of methane holes. This provides an additional level of complexity with this work as slots between approximately 38 and $44 \mathrm{~mm}$ are difficult to machine accurately.

The trends seen experimentally broadly agree with those calculated by the FE simulations. Both methods have a comparable localisation of the defect signal with coil angle and both demonstrate a trend between defect size and the magnitude of the phase change. 


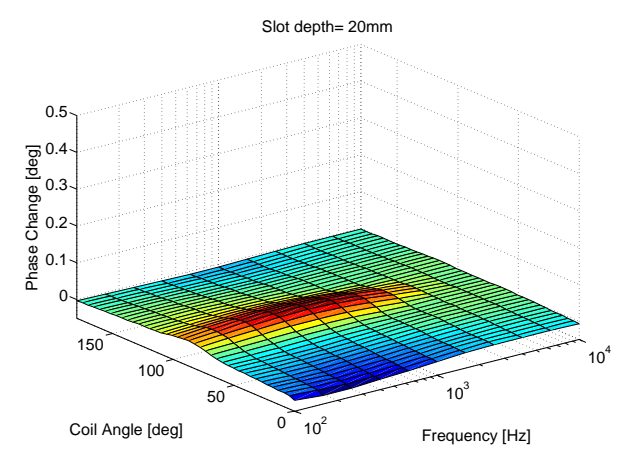

(a) $20 \mathrm{~mm}$ slot

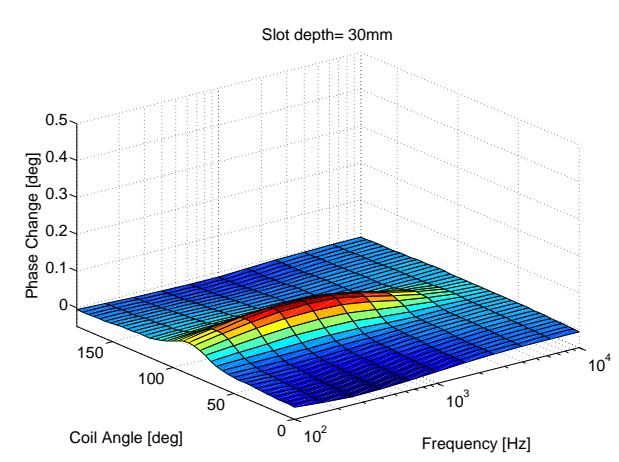

(b) $30 \mathrm{~mm}$ slot

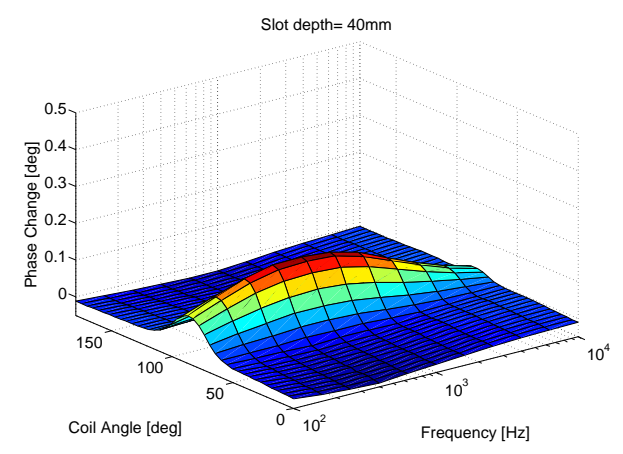

(c) $40 \mathrm{~mm}$ slot

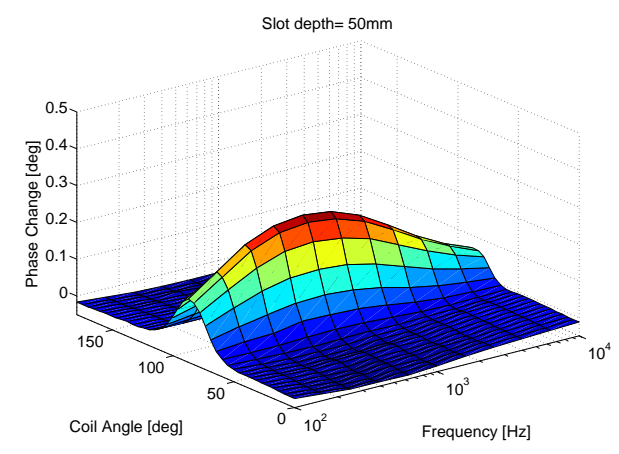

(d) $50 \mathrm{~mm}$ slot

Figure 6: Phase change measured for the $120 \mathrm{~mm}$ sensor with respect to coil position.

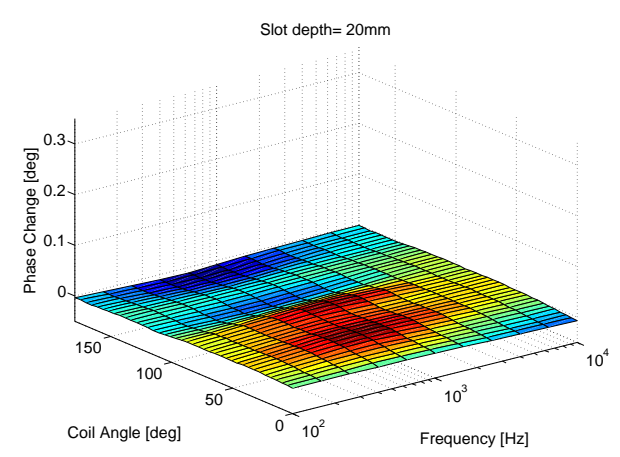

(a) $20 \mathrm{~mm}$ slot

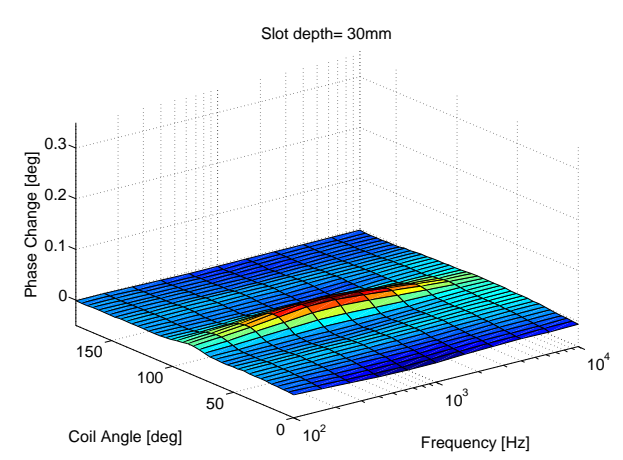

(b) $30 \mathrm{~mm}$ slot

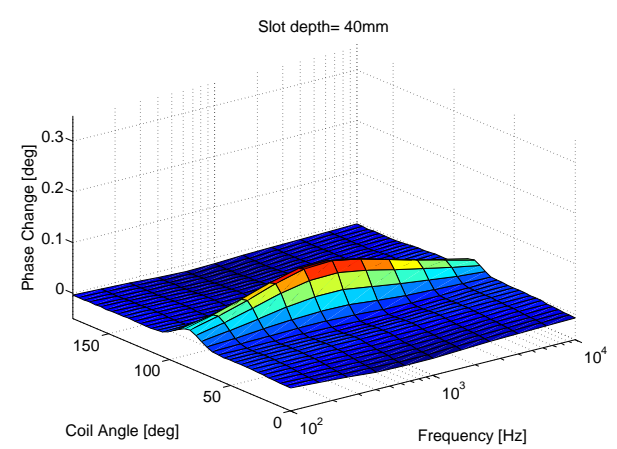

(c) $40 \mathrm{~mm}$ slot

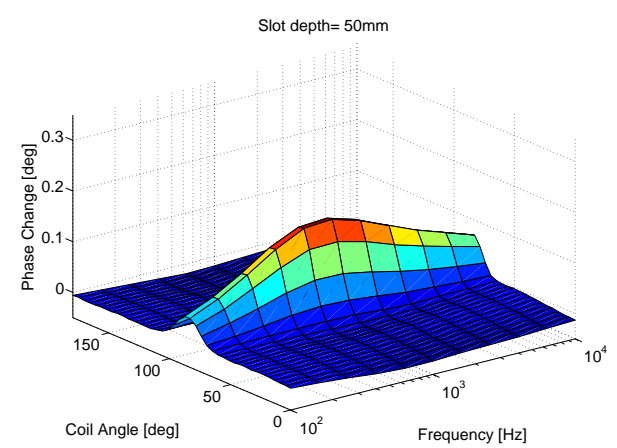

(d) $50 \mathrm{~mm}$ slot

Figure 7: Phase change measured for the PECIT sensor with respect to coil position. 


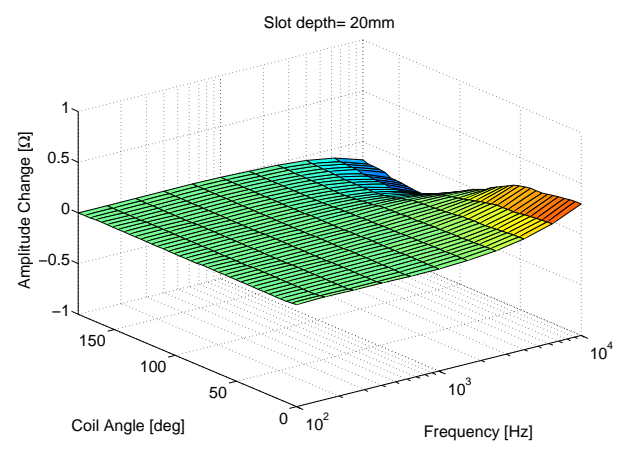

(a) $20 \mathrm{~mm}$ slot

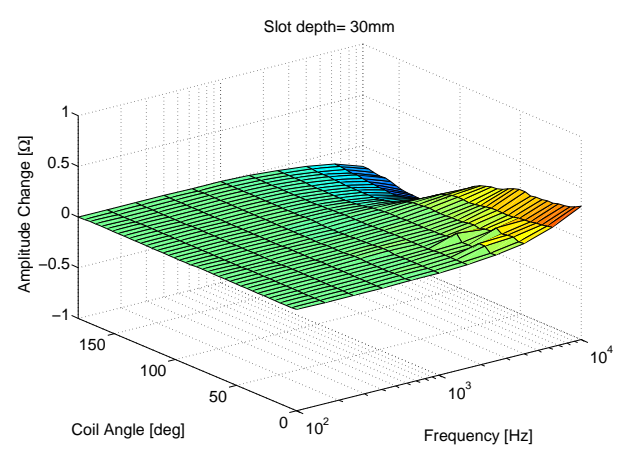

(b) $30 \mathrm{~mm}$ slot

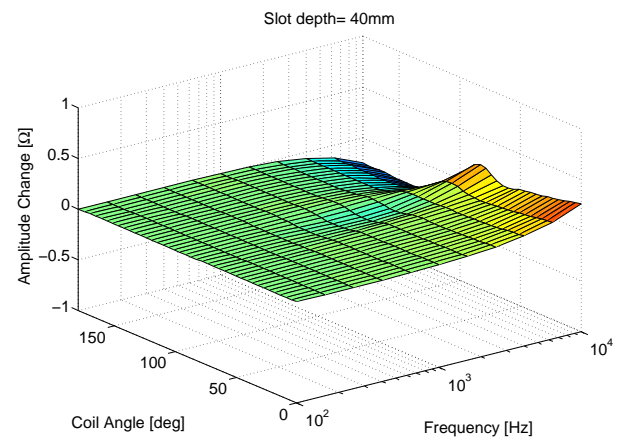

(c) $40 \mathrm{~mm}$ slot

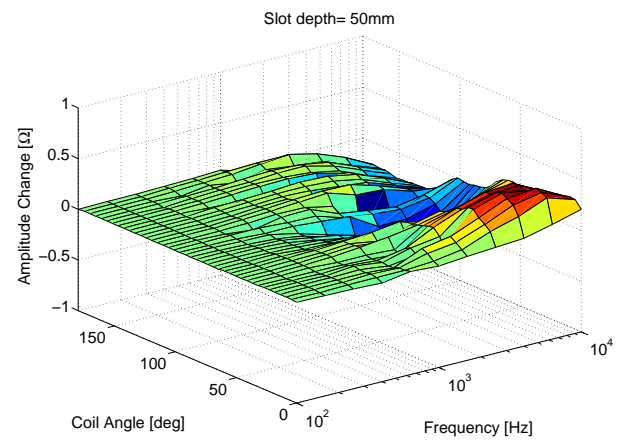

(d) $50 \mathrm{~mm}$ slot

Figure 8: Amplitude change measured for the $120 \mathrm{~mm}$ sensor with respect to coil position.

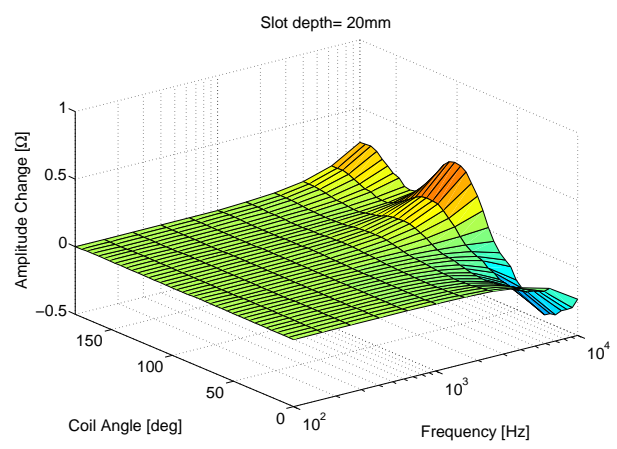

(a) $20 \mathrm{~mm}$ slot

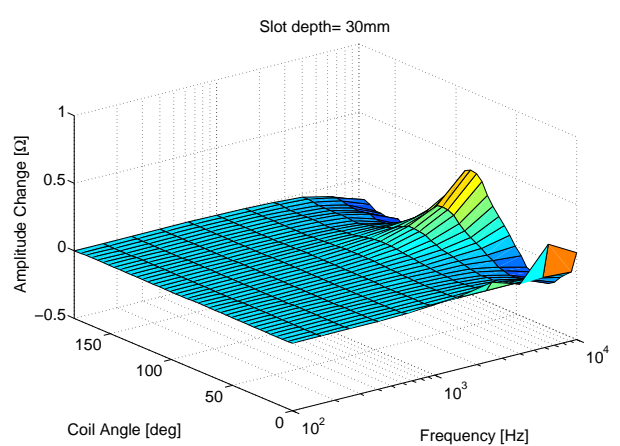

(b) $30 \mathrm{~mm}$ slot

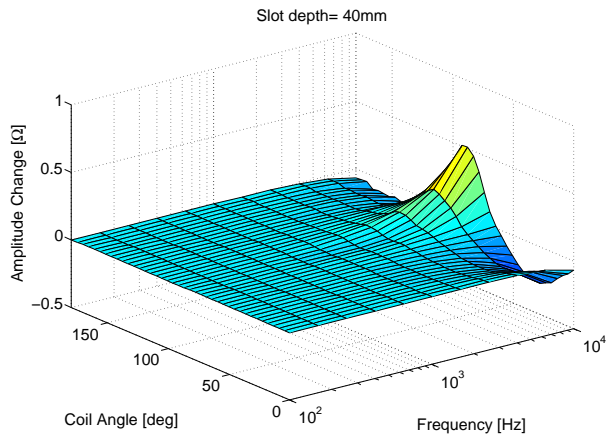

(c) $40 \mathrm{~mm}$ slot

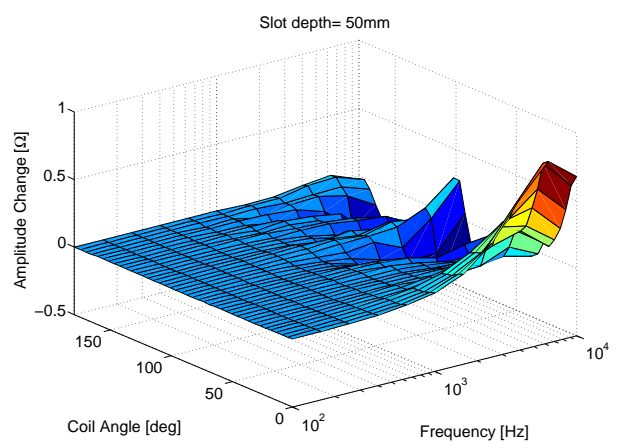

(d) $50 \mathrm{~mm}$ slot

Figure 9: Amplitude change measured for the PECIT sensor with respect to coil position. 


\begin{tabular}{|c|c|c|}
\hline Nominal slot size $[\mathrm{mm}]$ & $120 \mathrm{~mm}$ sensor & PECIT sensor \\
\hline $30 \mathrm{~mm}$ defect & 30 & 28 \\
\hline $50 \mathrm{~mm}$ defect & 50 & 50 \\
\hline
\end{tabular}

Table 3: Estimated slot size from experimental data.

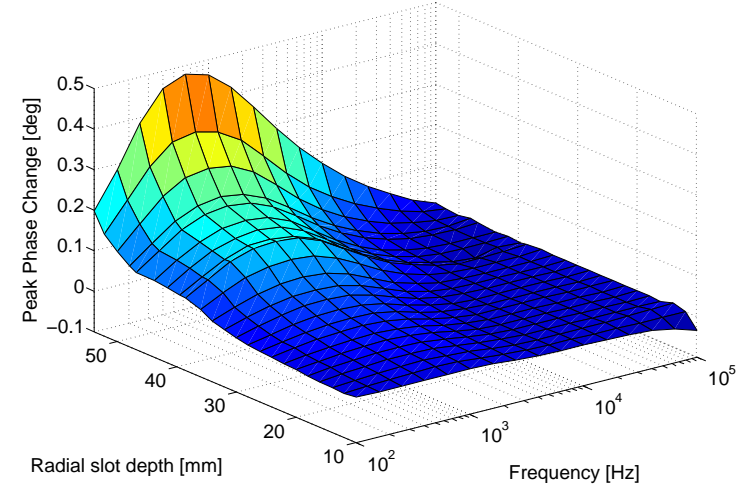

(a) $120 \mathrm{~mm}$ sensor

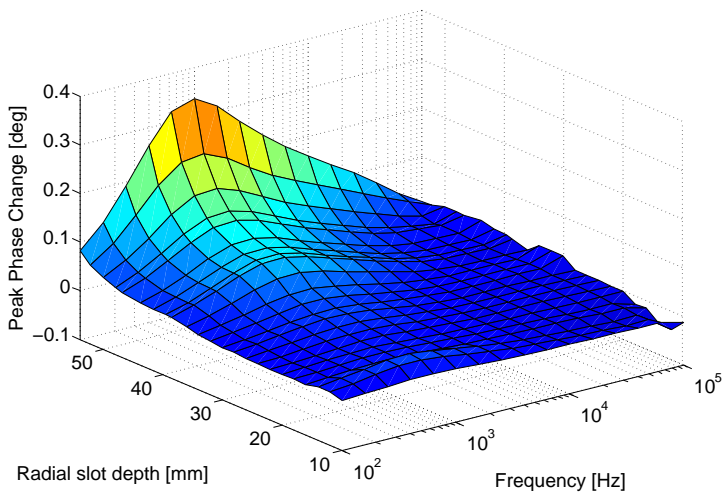

(b) PECIT sensor

Figure 10: Maximum phase changes measured for the two sensors used with respect to the measured slot size.

\subsection{Crack Sizing}

The (possibly large) variability in graphite density (and therefore conductivity) during manufacture introduces an additional level of complexity for this work. Very few pre-characterisation measurements were made before the AGRs were constructed and so a methodology which requires a reference dataset based on "virgin" graphite to estimate the position or size of a crack is of limited utility. In order to overcome this, a procedure was developed whereby each brick could act as its own reference. This was achieved by calculating the mean phase response of the brick over the range of coil positions being inspected. This gave a reference dataset against which local deviations (due to effects such as a subsurface slot) could be resolved.

This dataset can now be used to make estimates of a keyway root slot of unknown size. By making a positional and frequency sweep of the sensor past this defect the difference between the response surface for the defect and those obtained for known slot sizes can be minimised. The subtraction of the positional average at each frequency should remove the effects due to a brick having a potentially different bulk conductivity from the reference brick.

Additional brick sections were available allowing the validity of this method to be assessed. Two bricks were available which contained defects of 30 and $50 \mathrm{~mm}$ through wall extent (which is assumed to be initially unknown). Multi-frequency positional sweeps were performed on these bricks using the two sensors. A sample of the differences measured between the phase changes for a slot of unknown size and those for the slots of known slot size are shown in Figures 11 to 14 . The surface of minimal norm can now be selected and is assumed to correspond to the unknown defect size. The results of this are shown in Table 3.

\section{Conclusions}

This work reports the results of modelling and experimental programmes to assess subsurface cracking in AGR graphite. A finite element model which can calculate the phase response due to a subsurface defect has been described. This model has been shown to give an approximately smooth response with both frequency and coil position despite the fact that remeshing noise presents a significant barrier to accurate calculation. This remeshing results in the deviations from smoothness seen in Figure 4. However, the model does allow the location of slots to be determined and cracks of smaller radial extent give a phase response which is comparable to that measured experimentally once deviations from smoothness are taken 


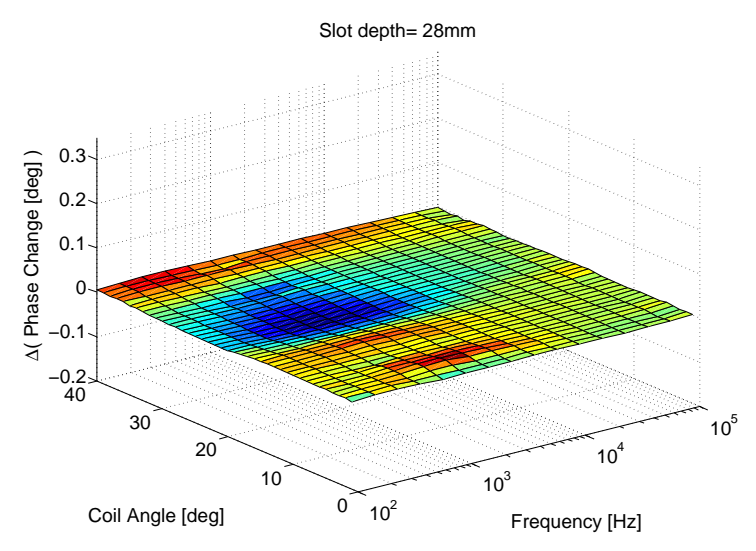

(a) $28 \mathrm{~mm}$ reference slot

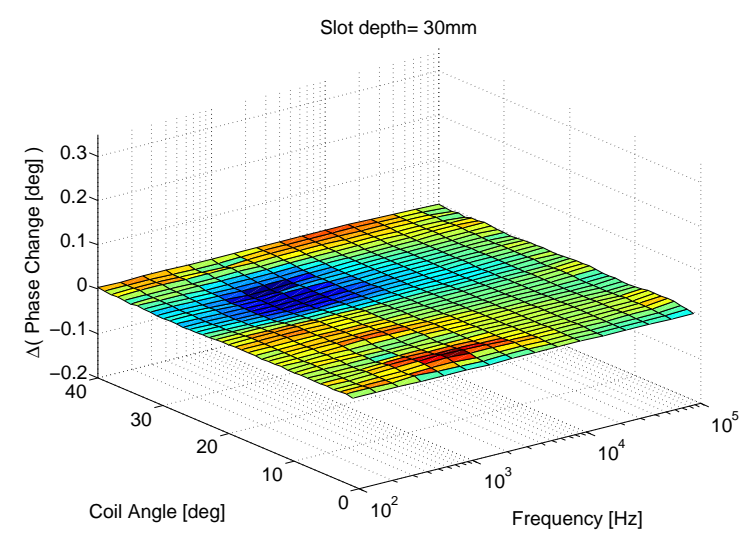

(b) $30 \mathrm{~mm}$ reference slot

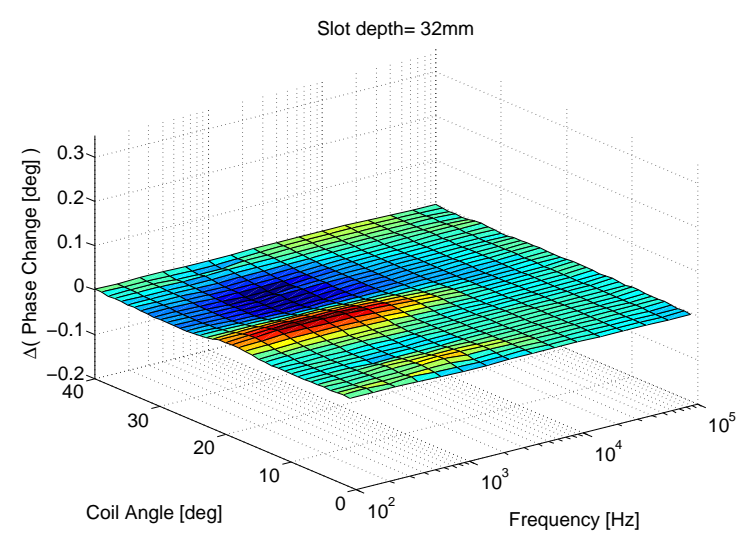

(c) $32 \mathrm{~mm}$ reference slot

Figure 11: The phase change for a slot of undetermined (30 mm) size subtracted from the measured phase change of the slots sizes shown. The resulting surface with the smallest norm is assumed to correspond to the correct slot size. This data was measured using the $120 \mathrm{~mm}$ sensor.

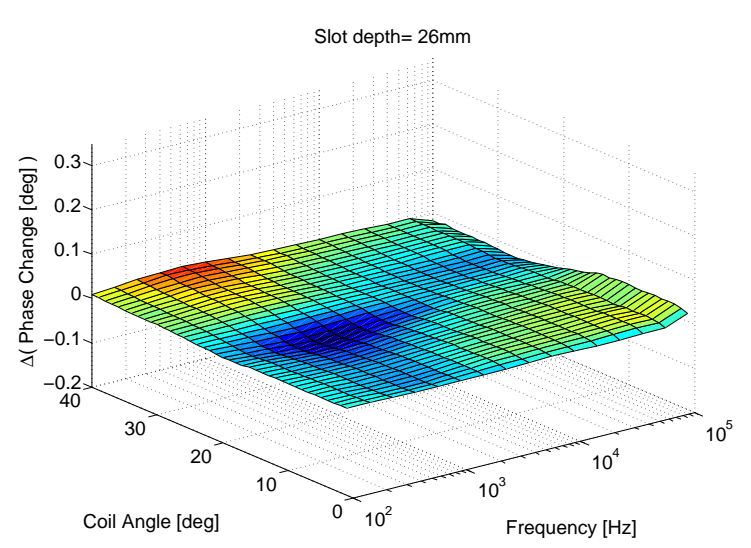

(a) $26 \mathrm{~mm}$ reference slot

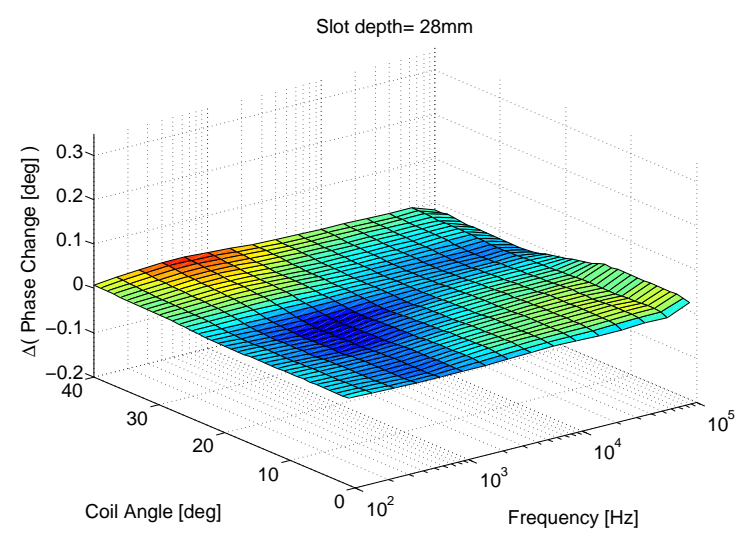

(b) $28 \mathrm{~mm}$ reference slot

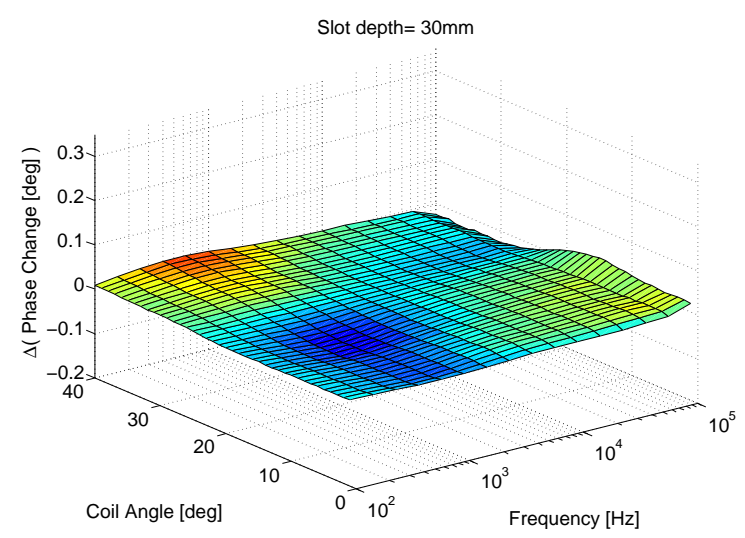

(c) $30 \mathrm{~mm}$ reference slot

Figure 12: The phase change for a slot of undetermined size $(30 \mathrm{~mm})$ subtracted from the measured phase change of the slots sizes shown. This data was measured using the PECIT sensor. 


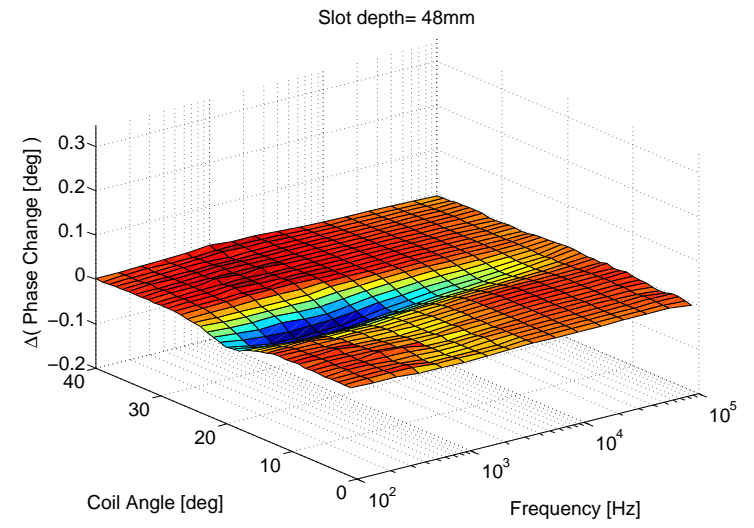

(a) $48 \mathrm{~mm}$ reference slot

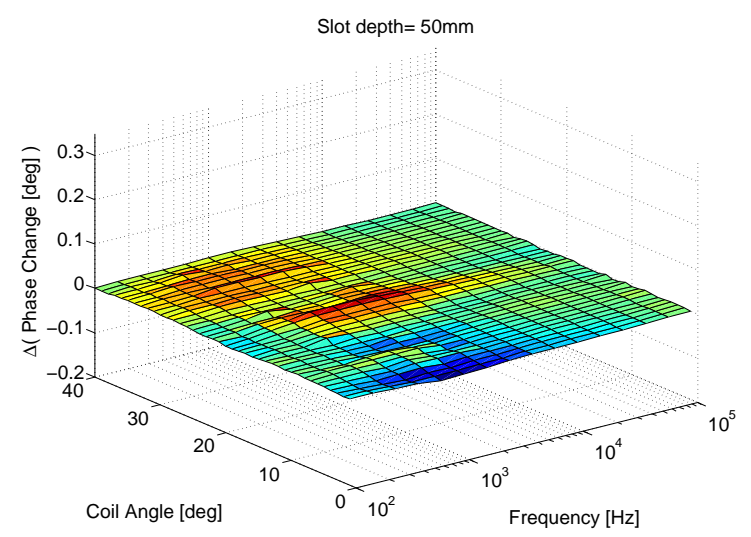

(b) $50 \mathrm{~mm}$ reference slot

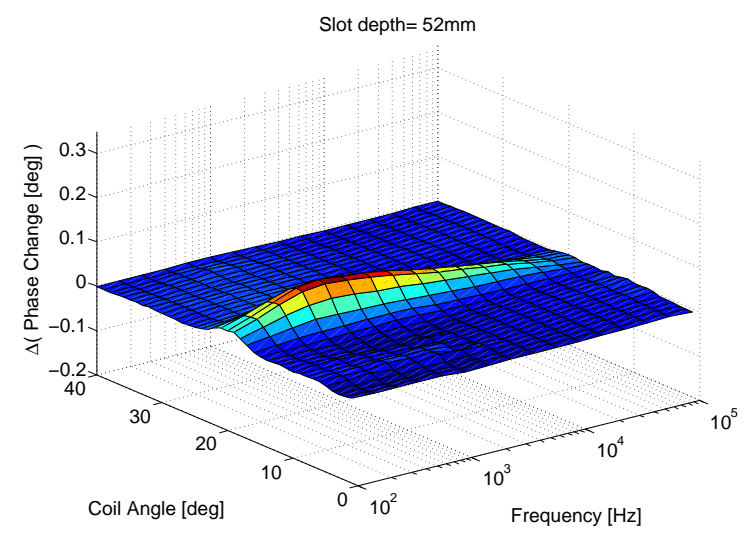

(c) $52 \mathrm{~mm}$ reference slot

Figure 13: The phase change for a slot of undetermined size $(50 \mathrm{~mm})$ subtracted from the measured phase change of the slots sizes shown. This data was measured using the $120 \mathrm{~mm}$ sensor.

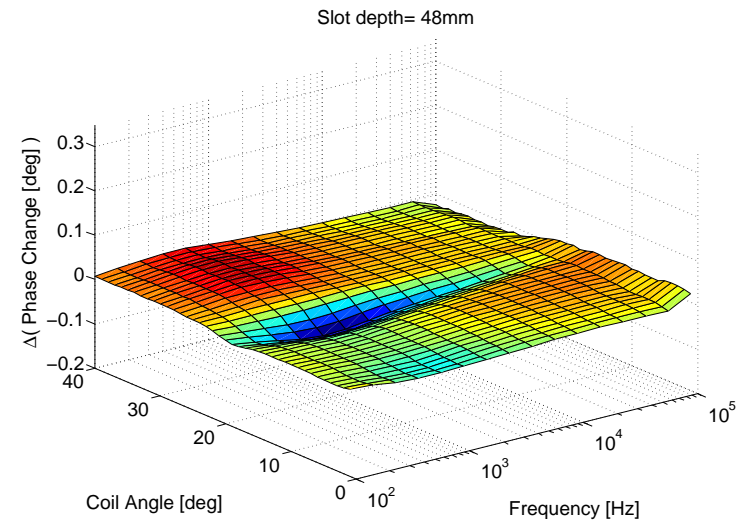

(a) $48 \mathrm{~mm}$ reference slot

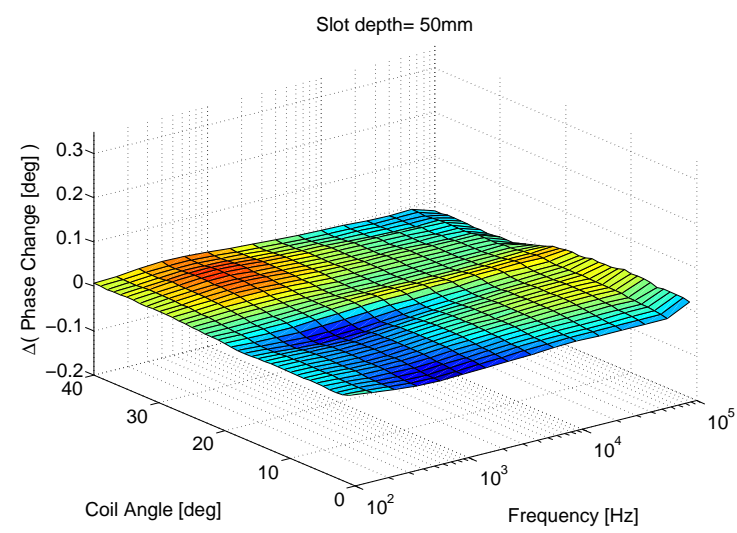

(b) $50 \mathrm{~mm}$ reference slot

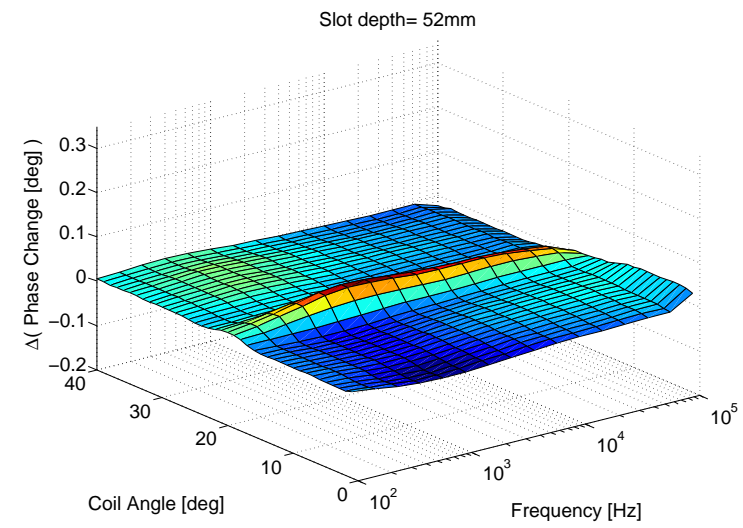

(c) $52 \mathrm{~mm}$ reference slot

Figure 14: The phase change for a slot of undetermined size $(50 \mathrm{~mm})$ subtracted from the measured phase change of the slots sizes shown. This data was measured using the PECIT sensor. 
into account. Both the experimental and modelling work show the expected trend between phase change and slot size, namely that smaller slots result in a smaller phase change.

Further refinement of the mesh used in the modelling should help to remove the deviations which occur and is an area of ongoing work.

It has been shown that subsurface defects in graphite can be detected and located with good accuracy using the experimental procedure described, provided they have an extent greater than approximately $10 \mathrm{~mm}$ from the base of the keyway. The phase changes due to the defects are spatially localised and allow the position of a slot to be determined. A method to estimate the size of such a defect has also been outlined. This is an empirical method which allows some of the variable material properties to be taken into account. The positional averaging procedure which has been described removes some of the effect due to the unknown bulk graphite conductivity and should improve the utility of the method for irradiated bricks. This has been tested on two additional samples which have a different bulk conductivity; however all the samples considered to date are virgin graphite. Reactor bricks have undergone significant irradiation and due to radiolytic oxidation they may have a conductivity which could be less than half that of the bricks presented in this study. Such a significant deviation in bulk conductivity may have a significant impact on the detectability of small slots.

Clearly, in a realistic scenario if a brick were to crack, stresses from surrounding bricks could force the crack to fully or partially close. This work has not addressed this due to the difficulty of precisely controlling the size of a genuine crack in a graphite sample of this geometry. Additional work is ongoing to assess the detectability of genuine subsurface cracks, although this is presently limited to a flat plate geometry.

Whilst this work has focused on graphite, the methodology described here should be applicable to a broad range of non-magnetic materials with appropriate choices of coil geometry and excitation frequency.

\section{Acknowledgments}

The authors would like to thank EDF Energy and the UK Engineering and Physical Sciences Research Council (EPSRC) via the Nuclear EngD Programme for their support of this research.

\section{References}

[1] Yin, W., Peyton, A.J.. Thickness Measurement of NonMagnetic Plates Using Multi-Frequency Eddy Current Sensors. NDT \& E International 2007;40(1):43 - 48. URL: http://www.sciencedirect.com/science/article/ B6V4C-4KXDR3S-1/2/ea9a08521569679eccf99d961a9f34ed. doi:DOI : 10.1016/j.ndteint.2006.07.009.
[2] Vasic, D., Bilas, V., Ambrus, D.. Pulsed eddy current nondestructive testing of ferromagnetic tubes. In: Instrumentation and Measurement Technology Conference, 2003. IMTC '03. Proceedings of the 20th IEEE; vol. 2. 2003, p. 1120-1125 vol.2. doi:10.1109/IMTC. 2003.1207928.

[3] McLachlan, N., Reed, J., Metcalfe, M.P.. AGR Core Safety Assessment Methodologies. In: Specialists Meeting on Graphite Moderator Lifecycle Behaviour. Bath, UK: IAEA; 1995, p. 125-136. URL: http://www.iaea.org/inisnkm/nkm/aws/htgr/ abstracts/abst_28008802.html.

[4] Brown, M., Greenaway, A., Williams, J.. Eddy Current Technique Development: Summary of Initial Survey Results from AGR Reactor Cores. In: 11th International Nuclear Graphite Specialists Meeting. Eastbourne, UK; 2010,

[5] Hur, D.H., Lee, D.H., Choi, M.S., Lee, U.C., Kim, S.J., Han, J.H.. Discrimination method of through-wall cracks in steam generator tubes using eddy current signals. NDT \& E International 2006;39(5):361 - 366. URL: http://www.sciencedirect.com/science/ article/pii/S0963869505001532. doi:http://dx.doi.org/10. 1016/j.ndteint.2005.10.005.

[6] Yusa, N., Machida, E., Janousek, L., Rebican, M., Chen, Z., Miya, K.. Application of eddy current inversion technique to the sizing of defects in inconel welds with rough surfaces. Nuclear Engineering and Design 2005;235(14):1469 - 1480. URL: http://www.sciencedirect.com/science/ article/pii/S002954930500049X. doi:http://dx.doi.org/10. 1016/j.nucengdes. 2005.01.005.

[7] Chady, T., Enokizono, M., Sikora, R., Todaka, T., Tsuchida, Y.. Natural crack recognition using inverse neural model and multi-frequency eddy current method. Magnetics, IEEE Transactions on 2001;37(4):2797-2799. doi:10.1109/20.951310.

[8] Fukutomi, H., Takagi, T., Nishikawa, M.. Remote field eddy current technique applied to nonmagnetic steam generator tubes. NDT \& E International 2001;34(1):17 - 23. URL: http://www.sciencedirect. com/science/article/pii/S0963869500000268. doi:http: //dx.doi.org/10.1016/S0963-8695(00)00026-8.

[9] Vernon, S.N.. Parametric eddy current defect depth model and its application to graphite epoxy. NDT International 1989;22(3):139 - 148. URL: http://www.sciencedirect.com/ science/article/pii/0308912689900011. doi:http://dx.doi. org/10.1016/0308-9126(89)90001-1.

[10] Lane, S., Moore, R., Groger, H., Gandhe, G., Griffin, O.. Eddy current inspection of graphite/epoxy laminates. Journal of Reinforced Plastics and Composites 1991;10(2):158-166. URL: http://jrp.sagepub.com/content/ 10/2/158.abstract. doi:10.1177/073168449101000204. arXiv:http://jrp. sagepub.com/content/10/2/158.full.pdf+html.

[11] Nateq, M.H., Kahrobaee, S., Kashefi, M.. Use of eddy-current method for determining the thickness of induction-hardened layer in cast iron. Metal Science and Heat Treatment 2013;55(78):370-374.

[12] Kunerth, D., McJunkin, T.R.. Nondestructive evaluation of nuclear-grade graphite. In: Review of Progress in Quantitative Nondestructive Evaluation; vol. 1430 of AIP Conf. Proc. American Institute of Physics; 2012, p. 1609-1616. 